\title{
Impact of a Digital Activity Tracker-based Workplace Activity Program on Health and Wellbeing
}

\author{
David G. Glance \\ The University of Western Australia \\ 35 Stirling Highway, Crawley \\ Western Australia, 6018 \\ +61864884706 \\ david.glance@uwa.edu.au
}

\author{
Esther Ooi \\ The University of Western Australia \\ 35 Stirling Highway, Crawley \\ Western Australia, 6018 \\ +61892240259 \\ esther.ooi@uwa.edu.au
}

\author{
Ye'elah Berman \\ The University of Western Australia \\ 35 Stirling Highway, Crawley \\ Western Australia, 6018 \\ +61864884706 \\ yeelah.berman@uwa.edu.au
}

\author{
Charlotte F. Glance \\ The University of Western Australia \\ 35 Stirling Highway, Crawley \\ Western Australia, 6018 \\ +61864884706 \\ charlotte.glance@uwa.edu.au
}

\author{
P. Hugh R. Barrett \\ The University of Western Australia \\ 35 Stirling Highway, Crawley \\ Western Australia, 6018 \\ +61864883459 \\ hugh.barrett@uwa.edu.au
}

\begin{abstract}
Chronic disease is endemic within the Australian community. 3.6 million Australians have diabetes or pre-diabetes with the number increasing by $7 \%$ each year. Fifty three percent of Australians have one or more chronic diseases. Increasing levels of activity has proved relatively straightforward, especially through workplace physical activity interventions. What is still not certain are the short, and long-term, health benefits arising from these workplace activity challenges. Research into workplace activity challenges is beset with a number of methodological obstacles that may, in part, explain why consistent outcomes have not been found from studies of this type. The aim of this study was to assess whether participation in a 16-week activity challenge would result in measurable changes in lipid profile, blood glucose, renal function, blood pressure, weight and health and wellbeing as measured using a health and wellbeing assessment. The study demonstrated that participants could increase their levels of activity and maintain at least 10,000 steps a day for a period of 16 weeks. The study also identified that participants in teams were significantly more active than those participating as individuals. Furthermore, attrition from the activity challenge was greater amongst participants not in a team. This demonstrated the importance of social interactions, support and possibly other factors that being part of a group brought to the experience of participating in the activity challenge. In addition to the above, the challenge resulted in reductions in non-HDL cholesterol, and triglyceride concentrations and health and wellbeing score.
\end{abstract}

\section{CCS Concepts}

- Applied Computing $\rightarrow$ Life and Medical Sciences $\rightarrow$ Bioinformatics - Human Centered Computing $\rightarrow$ Collaborative and Social Computing Systems and Tools

\footnotetext{
Permission to make digital or hard copies of part or all of this work for personal or classroom use is granted without fee provided that copies are not made or distributed for profit or commercial advantage and that copies bear this notice and the full citation on the first page. Copyrights for third-party components of this work must be honored.

Copyright is held by the owner/author(s).

DH'16, April 11-13, 2016, Montréal, QC, Canada.

ACM 978-1-4503-4224-7/16/04.

Doi: http://dx.doi.org/10.1145/2896338.2896345

(c) (i)

W is licensed under a Creative Commons Attribution 4.0

International License.
}

\section{Keywords}

Public Health; Workplace Activity Program; Wearable Activity Tracker; Digital Pedometer; Digital Activity Tracker; Chronic Disease; Health and Wellbeing; Physical Activity.

\section{INTRODUCTION}

Chronic disease is endemic within the Australian community. 3.6 million Australians have diabetes or pre-diabetes ${ }^{1}$ with the number increasing by $7 \%$ each year. Fifty three percent of Australians have one or more chronic diseases [1]. Mental illness is also extremely prevalent with $20 \%$ of Australians surveyed by the Australian Bureau of Statistics experiencing symptoms of a mental disorder in the 12 months prior to a survey carried out in 2007 [2].

At the organisational level, the cost of chronic disease can be significant. In one study in the US, the impact was a cost of $11 \%$ of total labour costs, with $7 \%$ being attributable to "presenteeism" - staff turning up to work when they are sick [3]. The impact of chronic disease on staff productivity may be more extensive, however, as the impact of staff who are suffering have on others, in terms of decreased productivity and increased absenteeism is difficult to measure.

The largest contributing factor to the prevalence of chronic disease is lifestyle. Weight is a major issue with $27.5 \%$ of the Australian population being obese [4]. Diet, smoking, alcohol, and lack of physical activity (PA) all contribute to increased morbidity due to chronic diseases. Lifestyle factors are by definition, something that people can change and something that it is in the commercial and social interest of organisations to help promote and support.

Increasing levels of activity has proved relatively straightforward, especially through workplace PA interventions [5]. These interventions have been codified into "workplace activity challenges (WAC)" involving staff forming teams and maintaining 10,000 steps a day for a period of 12 - 16 weeks [6]. Physical activity can be measured through electronic pedometers

\footnotetext{
${ }^{1}$ https://www.diabetesaustralia.com.au
} 
such as those manufactured by companies like Fitbit ${ }^{2}$, Jawbone ${ }^{3}$ and Misfit ${ }^{4}$. The steps are automatically uploaded and made available through the challenge software.

What is still not certain are the short, and long-term, health benefits arising from these workplace activity challenges. In a review of the literature, To et al [5] found that most studies reported increases in PA and a reduction in BMI. Other studies and reviews have demonstrated reductions in systolic and diastolic blood pressure [7, 6], decreased waist circumference and increased vegetable and fruit intake [6]. Interestingly, Freak-Poli et al found an increase in fasting total cholesterol and triglycerides [6]. These changes persisted at least for a further 8 months after the challenge ended [8]. Finally, workplace activity challenges have been associated with an improvement in the mental component scores of health related quality of life assessments [9].

Research into workplace activity challenges is beset with a number of methodological obstacles that may, in part, explain the inconsistent outcomes found from studies of this type. Firstly, the group of people that undertake these challenges are self-selected and their motivation to participate may range from feeling pressured to participate by work colleagues or employers, through to losing weight and improving general health and wellbeing. Secondly, there is normally a high attrition rate of participants in these challenges, further distinguishing the population of people being studied. In one study of participants undertaking 4-month activity challenge, there was an attrition of $30 \%$ with significant differences between the returning and non-returning participant groups [9]. Another factor is that workplace activity challenge participants are more commonly female.

The aim of this study was to assess whether participation in a 16week activity challenge would result in measurable changes in lipid profile, blood glucose, renal function, blood pressure, weight and health and wellbeing as measured using a health and wellbeing assessment. In order to run the activity challenge, a system called Self eHealth was developed for mobile and web to run both the activity challenge and manage the collection of activity and health and wellbeing data.

\section{METHODS}

\subsection{Study Population}

Participants were recruited from staff members at the University of Western Australia (UWA) through the UWA Human Resources Newsletter on health and safety, social media accounts and mail outs. Three hundred and fifty-three staff members enrolled in the activity challenge component of the study and were eligible for subsidized Fitbit devices from UWA. Of these, 111 participated in a health and wellbeing (HWB) assessment, which included completing a questionnaire, anthropometric measurements and biochemical assessments. All participants provided written informed consent. The UWA Human Research Ethics Committee approved the study protocol.

\subsection{Study Design}

The study was carried out over a 16 -week period between $25^{\text {th }}$ May and $27^{\text {th }}$ September 2015 . Participants were asked to walk,

\footnotetext{
${ }^{2}$ http://www.fitbit.com

${ }^{3}$ https://jawbone.com/

${ }^{4} \mathrm{http}: / /$ misfit.com/
}

run or engage in other exercise to achieve an equivalent 10,000 step a day target as part of the study. Participants could either undertake the study as part of a team or as an individual.

\subsection{Self eHealth Platform}

The activity challenge was managed through a purpose-built platform called Self eHealth. Self eHealth was made available to challenge participants via a website and Android and Apple apps. The Self eHealth application managed different aspects of the activity challenge as well as the on-boarding and data collection aspects of the research project.

Figure 1 outlines the different functional components of Self eHealth. Participants were able to connect their Fitbit (or Jawbone) devices to the app to allow for automated data collection. Summary pages provided detailed updates of progress that included the current day's activity as well as a series of progress averages, displayed as data and graphs. A second component concentrated on "Gamification" aspects by providing leaderboards, badges and information about weekly prizes. A different component integrated Instagram ${ }^{5}$ postings that were associated with the challenge. A separate Facebook $^{6}$ page provided participants with updates and information about the challenge. Regular social media events were created and staged for challenge members to participate in.

On the research side, the Self eHealth platform offered enrolment of research project participants. This provided project information and obtained consent for the project. The HWB assessment was provided through this functionality as an online form that could be completed through the web application or the mobile apps. Finally, data collected through blood tests was provided to the participants, as well as feedback about where these data fitted into normal expected ranges.

Feedback was sought from participants regarding the application interface and new functionality was added on a nightly basis as a result of this feedback. The feedback was very positive although the majority of participants chose to use the web application rather than the mobile apps.

The platform functionality is able to be permissioned in such a way as to personalize any given challenge and configure the research administration component with a great deal of flexibility.

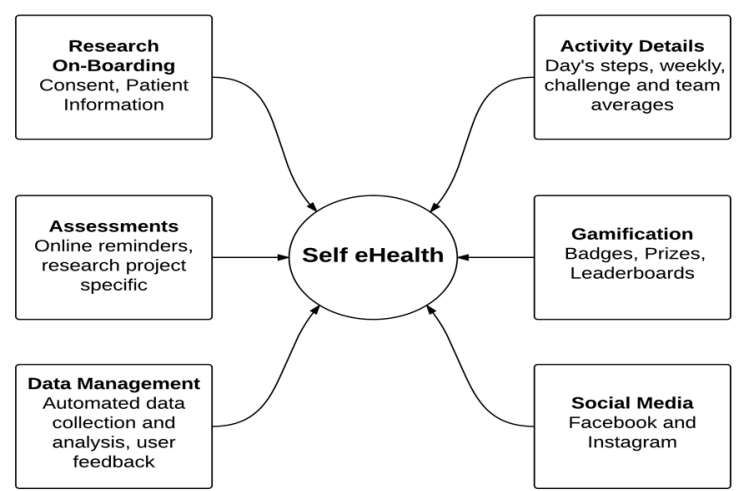

Figure 1: Self eHealth platform for managing activity challenge and research study.

\footnotetext{
${ }^{5}$ https://www.instagram.com/

${ }^{6}$ https://www.facebook.com/
} 


\subsection{Data Collection}

Data were collected online through the Self eHealth application. Step information was obtained on an hourly basis using the Fitbit $A P I$ together with the Self eHealth application over the 16-week period for all participants.

Participants involved in the health and well-being component had measurements taken at baseline and at the end of the 16-week period. Weight, height and blood pressure measurements were collected by trained staff and biochemical measurements were collected through venipuncture and assessed by an independent pathology practice (Clinipath Pathology ${ }^{7}$ ). Non-HDL cholesterol was calculated as total cholesterol minus HDL cholesterol. These participants also completed an online, 29-question health and wellbeing assessment that consisted of 20-question Vielife ${ }^{8}$ corporate health and wellbeing assessment together with the 9question Patient Health Questionnaire 9 [PHQ-9]). The 20question Vielife assessment provides a score out of 100 and has been validated against other accepted standard health and wellbeing instruments [10]. The PHQ-9 is scored out of 27 and has been validated in the general population against other measures of depression severity $[11,12]$.

\subsection{Statistical Analyses}

Analyses were performed using STATA (Version 12.1; StataCorp, College Station, TX). Data are presented as mean and standard error of the mean (SEM) unless specified otherwise. Skewed variables were logarithmically transformed where appropriate. To assess differences at baseline and at the end of the study a paired t-test was applied. To examine statistical associations between variables at baseline and changes in variables, simple linear regression methods were used. P-values are reported, with statistical significance set at the $5 \%$ level.

To determine whether the major components identified by the Vielife assessment were also observed in our cohort, factor analysis was performed. In brief, all 20 items were used. Components were determined by factor analysis using the method of principal component extraction and varimax rotation. The number of components identified was determined a priori. These include, eigenvalue $>1$ criterion, identification of a break point in the scree plot, interpretability and cumulative proportion of variance explained. Items were considered to load highly on a component if they had a factor loading $\geq 0.3$ with that component. Inter-item reliability was assessed by Cronbach's $\alpha$-coefficient. A coefficient of $\geq 0.7$ considered as acceptable.

\section{RESULTS}

\subsection{Retention}

Of the 353 participants who enrolled into the activity challenge, $68 \%$ participated within a team. There were 43 teams with a minimum size of two and a maximum of 19 participants. The average daily steps over the 16-week period for all participants was 9,671 (range 4,239-34,388) steps. The average daily steps for those that completed twelve or more weeks of the challenge was 10,542 , with a peak at week 3 of 11,468 steps falling to 9,688 steps per day at the end of the challenge (Figure 2).

\footnotetext{
${ }^{7}$ http://www.clinipathpathology.com.au/

${ }^{8} \mathrm{http}: / /$ www.vielife.com/
}

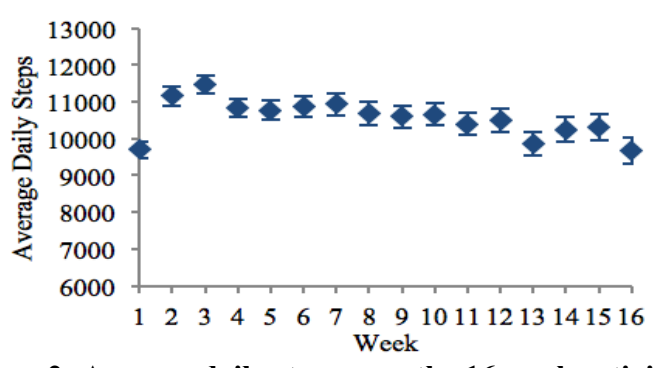

Figure 2: Average daily steps over the 16-week activity challenge.

The average daily steps over the 16 -week period for participants who were part of a team were 2,041 steps higher than those who participated as individuals $(10,317 \pm 287$ vs. 8,276 $\pm 317, \mathrm{p}<0.01$, Figure 3).

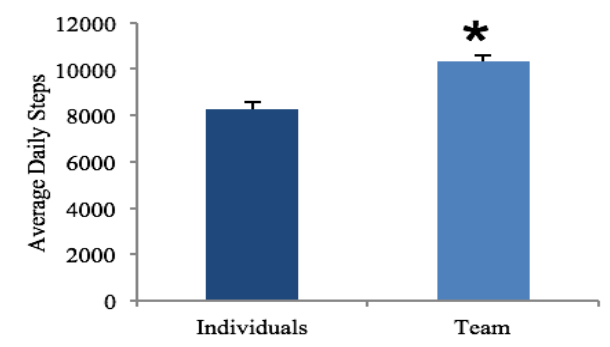

*Denotes $\mathrm{p}<0.05$

Figure 3: Average daily steps over the 16-week activity challenge in those who participated as individuals or as part of a team.

The dropout rate over the course of the 16 -weeks was $7 \%$ per 4 weeks. There was a lower dropout rate for those who participated as part of a team ( $6.5 \%$ vs. 9.6\% per 4 -weeks, $p<0.01$, Figure 4$)$.

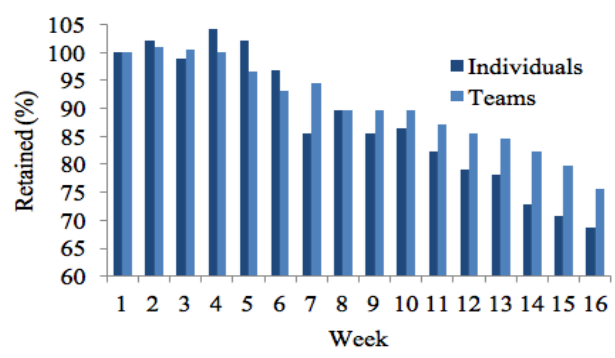

Figure 4: Retention over the 16-week activity challenge.

\subsection{Change Between Baseline and End of Challenge}

Eighty-one of 111 participants completed the health and wellbeing component. The average daily steps over the 16-week period in these participants were 11,046 (range 3,059-34,389) steps. The clinical and biochemical characteristics of the participants at baseline and at the end of the study are shown in Table 1. The average age of the participants was 42 (range $18-$ 68 ) years with $78 \%$ being female.

Participants maintained their average daily steps between baseline and end of challenge $(10,292 \pm 505$ vs. $10,386 \pm 659$ steps, $\mathrm{p}=0.87)$. At the end of the challenge, plasma triglyceride $(-14 \%$, Figure 5) and non-HDL cholesterol (-4\%, Figure 6) concentrations were significantly lower ( $\mathrm{p}<0.05$, for both). 
Table 1: Clinical and biochemical characteristic of study participants.

\begin{tabular}{lccc}
\hline & Week 0 & Week 16 & p \\
\hline Age (years) & $42 \pm 1.33$ & & \\
Weight $(\mathrm{kg})$ & $70.7 \pm 2.42$ & $70.6 \pm 2.34$ & 0.59 \\
BMI $\left(\mathrm{kg} / \mathrm{m}^{2}\right)$ & $24.7 \pm 0.66$ & $24.7 \pm 0.64$ & 0.62 \\
Systolic $(\mathrm{mmHg})$ & $116 \pm 2.11$ & $119 \pm 1.80$ & 0.05 \\
Diastolic $(\mathrm{mmHg})$ & $81.1 \pm 1.22$ & $81.1 \pm 1.46$ & 0.98 \\
Glucose $(\mathrm{mmol} / \mathrm{L})$ & $4.84 \pm 0.09$ & $4.89 \pm 0.08$ & 0.30 \\
TC $(\mathrm{mmol} / \mathrm{L})$ & $5.18 \pm 0.15$ & $5.04 \pm 0.12$ & 0.09 \\
TG $(\mathrm{mmol} / \mathrm{L})$ & $1.07 \pm 0.09$ & $0.92 \pm 0.08$ & $<0.01$ \\
HDL $(\mathrm{mmol} / \mathrm{L})$ & $1.73 \pm 0.08$ & $1.71 \pm 0.07$ & 0.47 \\
LDL $(\mathrm{mmol} / \mathrm{L})$ & $2.94 \pm 0.12$ & $2.91 \pm 0.13$ & 0.67 \\
Non-HDL $(\mathrm{mmol} / \mathrm{L})$ & $3.50 \pm 0.15$ & $3.30 \pm 0.15$ & 0.05 \\
Urea $(\mathrm{mmol} / \mathrm{L})$ & $4.39 \pm 0.20$ & $4.91 \pm 0.16$ & $<0.01$ \\
Creatinine $(\mathrm{mmol} / \mathrm{L})$ & $75.0 \pm 2.29$ & $75.7 \pm 2.47$ & 0.44 \\
Sodium $(\mathrm{mmol} / \mathrm{L})$ & $140 \pm 0.25$ & $140 \pm 0.30$ & 0.59 \\
Potassium $(\mathrm{mmol} / \mathrm{L})$ & $4.59 \pm 0.05$ & $4.58 \pm 0.05$ & 0.88 \\
Chloride $(\mathrm{mmol} / \mathrm{L})$ & $101 \pm 0.24$ & $101 \pm 0.29$ & 0.79 \\
Bicarbonate $(\mathrm{mmol} / \mathrm{L})$ & $26.3 \pm 0.26$ & $26.4 \pm 0.30$ & 0.77 \\
HWB score & $73.6 \pm 2.31$ & $78.4 \pm 2.70$ & 0.04 \\
PHQ9 score & $6.89 \pm 0.85$ & $2.07 \pm 0.68$ & 0.07 \\
\hline
\end{tabular}

Mean \pm SEM

To convert HDL cholesterol in $\mathrm{mmol} / \mathrm{L}$ to $\mathrm{mg} / \mathrm{dl}$, divide by 0.0259 ; triglycerides in $\mathrm{mmol} / \mathrm{L}$ to $\mathrm{mg} / \mathrm{dl}$, divide by 0.0113; TC: Total Cholesterol, TG: Triglycerides, HDL: high-density lipoprotein protein, LDL: low-density lipoprotein, Non-HDL cholesterol = $T C-H D L$

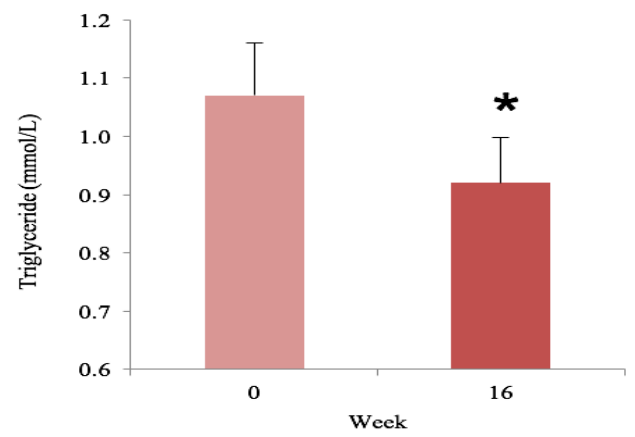

*Denotes $p<0.05$

Figure 5: Plasma triglyceride concentrations at baseline and at the end of the activity challenge.

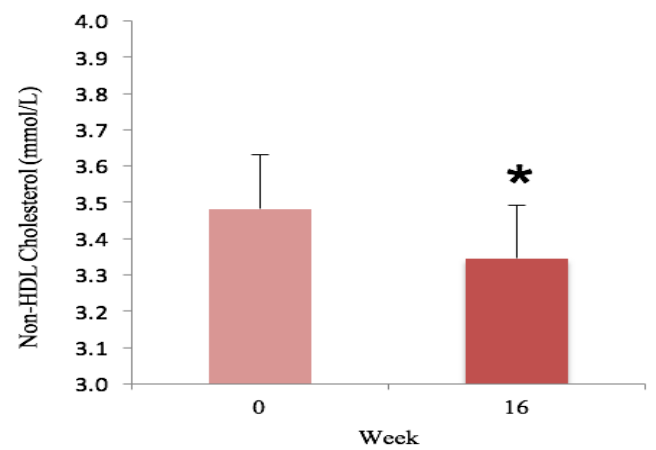

*Denotes $p<0.05$

Figure 6: Non-HDL cholesterol concentrations at baseline and at the end of the activity challenge.
A trend towards a reduction in total cholesterol $(-2 \%, \mathrm{p}=0.09)$ was also observed. These changes occurred without changes to body weight or BMI. There was a small but statistically significant increase in systolic blood pressure, but not diastolic blood pressure. The HWB score at the end of the challenge was 5 points higher compared with baseline $(73.6 \pm 2.31$ vs. $78.4 \pm 2.70$, $p=0.04$, Figure 7 ). This change was greater in participants who undertook the study as part of a team, but the differences were not statistically significant (Figure 8).

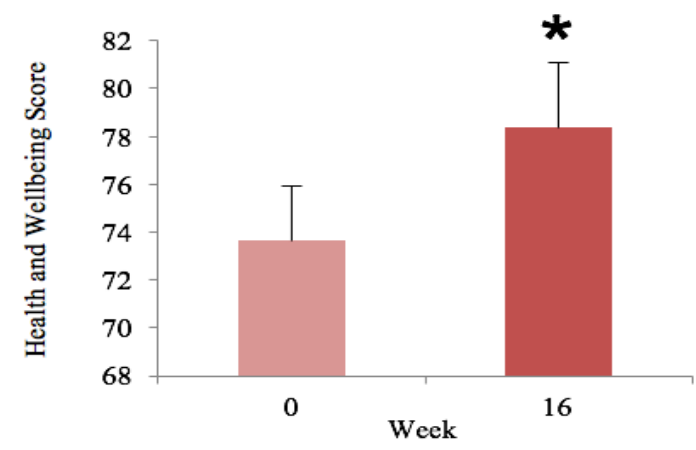

*Denotes $p<0.05$

Figure 7: Health and wellbeing score at baseline and at the end of the activity challenge.

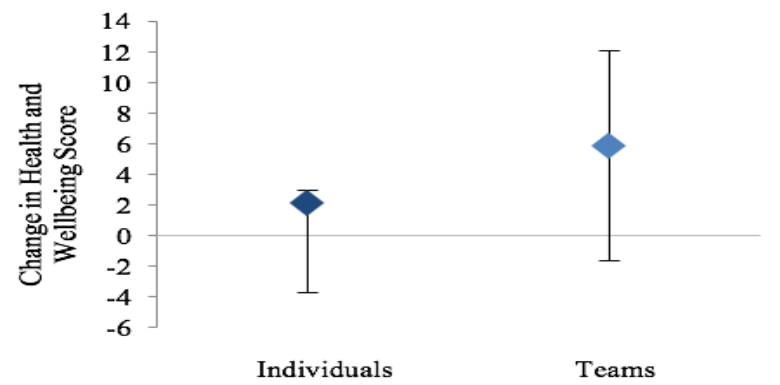

Figure 8: Change in health and wellbeing score in those who participated as individuals or as part of a team. Data shown as median and interquartile range.

\subsection{Associations}

At baseline, BMI was positively correlated with plasma total cholesterol, LDL cholesterol and triglyceride $(r=0.29, r=0.45$ and $r=0.33$, respectively) and negatively with HDL cholesterol concentration $(r=-0.34) \quad($ all $\mathrm{p}<0.05)$. BMI was negatively correlated with the HWB score $(r=-0.37, \mathrm{p}<0.01)$. Neither BMI $(r=-0.15, \mathrm{p}=0.20)$ nor the HWB score $(r=0.17, \mathrm{p}=0.16)$ were significantly correlated with the average daily steps at baseline. Change in HWB was significantly and positively correlated with the change in the number of steps between baseline and end of the challenge $(r=0.48, \mathrm{p}=0.02)$.

\subsection{Principle Component Analyses (PCA)}

The Vielife HWB assessment instrument identified 7 subscales for their population of corporate employees [10]. Our initial PCA produced seven components from the 20 health and wellbeing questions that were modelled. These seven components explained $67.5 \%$ of the variance in the population. However, only the stress subscale and the overall survey of 20 questions showed Cronbach's $\alpha$ coefficients above 0.7 , the normal threshold that implies consistency [13] (stress subscale Cronbach's $\alpha=0.74$, Overall HWB assessment Cronbach's $\alpha=0.72$ ), and so in the 
current project's assessment, the sub-scales could not be used independently. As a result, only the overall value for health and wellbeing was used and no attempt was made to use the subscales.

\section{DISCUSSION}

The current study demonstrated that most participants could increase their levels of activity and maintain at least 10,000 steps a day for a period of 16 weeks. The study also identified that participants in teams were significantly more active than those participating as individuals. Furthermore, attrition from the activity challenge was greater amongst participants not in a team. This demonstrated the importance of social interactions, support and possibly other factors that being part of a group brought to the experience of participating in the activity challenge.

In addition to the above, the challenge resulted in reductions in non-HDL cholesterol and triglyceride concentrations and an improved health and wellbeing score. The improvement in plasma lipids is a positive outcome, but is in contrast to the study of Freak-Poli et al [6] who showed an increase in these measures after an equivalent duration of activity. Other studies have shown variable changes to lipids. Whether the improvement in lipids seen in this study were a consequence of increased levels of physical activity or a concomitant change to improved diet requires further investigation.

The current study also did not show a change in BMI in contrast to other reported pedometer-based interventions [5]. The baseline measurements in this study did show the importance of BMI. It was positively correlated to HWB, total cholesterol, LDL cholesterol and triglyceride and negatively correlated with HDL cholesterol.

By their very nature, workplace activity challenges are going to select different, relatively small groups of people that may not be representative of the general population and this will, in all likelihood, affect the outcomes. Previously reported studies have varied by the nature of the intervention, with some focusing on weight, smoking and diet in addition to activity. Unravelling the effects of confounding variables is difficult in this type of study.

Our study has limitations: there was no control group with which to compare changes in risk factors and health and wellbeing, the challenge population was not representative of the general population, and the number of participants in the challenge was relatively small. Future studies should address such limitations.

Activity challenges are an effective means of getting large groups of people to increase and maintain a higher level of activity that brings real health benefits. To be effective at a broader level, activity challenges need to involve a majority of the population they are offered to, in order to get beyond the relatively small percentage of the workforce that they currently involve. Different incentives may need to be applied depending on the motivational profiles of the larger group. Once involved in an activity challenge, maintaining daily targets and extending these to bring about greater health benefits will also need a range of approaches, the nature and effectiveness of which will be the subject of future research.

\section{ACKNOWLEDGEMENTS}

Hugh Barrett is a research fellow of the National Health and Medical Research Council of Australia. Esther Ooi is a Fellow of the Heart Foundation (Award ID: 100422)

\section{REFERENCES}

[1] Australian Institute of Health and Welfare. AIHW. Chronic diseases and associated risk factors in Australia, 2006. Canberra, ACT; 2006.

[2] Slade, J., Teesson, W., and Burgess, P. 2009. The mental health of Australians 2: report on the 2007 National Survey of Mental Health and Wellbeing.

[3] Collins, J. J., Baase, C. M., Sharda, C. E., Ozminkowski, R. J., Nicholson, S., Billotti, G. M., ... \& Berger, M. L. (2005). The assessment of chronic health conditions on work performance, absence, and total economic impact for employers. Journal of Occupational and Environmental Medicine, 47(6), 547-557.

[4] Australian Bureau of Statistics. 2013. Australian Health Survey: Updated Results, 2011-12, 'Table 5.1 Body Mass Index (a), Persons (estimate), Relative Standard Error of estimate, Proportion of persons, Relative Standard Error of proportion and 95\% Margin of Error - Australia', non-age standardised rates, cat. no. 4364.0.55.003, Retrieved on March 2015,

[5] To, Q. G., Chen, T. T., Magnussen, C. G., and To, K. G. 2013. Workplace physical activity interventions: a systematic review. Health Promotion. 27, 6 (Aug. 2013), e113-e12.

[6] Freak-Poli, R., Wolfe, R., Backholer, K., De Courten, M., and Peeters, A. 2011. Impact of a pedometer-based workplace health program on cardiovascular and diabetes risk profile. Preventive medicine. 53, 3 (Sept. 2011), 162171.

[7] Bravata, D. M., Smith-Spangler, C., Sundaram, V., Gienger, A. L., Lin, N., Lewis, R., ... and Sirard, J. R. 2007. Using pedometers to increase physical activity and improve health: a systematic review. JAMA. 298, 19 (Nov. 2007), 22962304.

[8] Freak-Poli, R., Wolfe, R., Brand, M., Courten, M., and Peeters, A. 2013. Eight-month postprogram completion: Change in risk factors for chronic disease amongst participants in a 4-month pedometer-based workplace health program. Obesity. 21, 9 (Sept. 2013), E360-E368.

[9] Harding, J., Freak-Poli, R. L. A., Backholer, K., and Peeters, A. 2013. Change in health-related quality of life amongst participants in a 4-month pedometer-based workplace health program. J Phys Act Health, 10, 4. 533-43.

[10] Mills, P. R. 2005. The development of a new corporate specific health risk measurement instrument, and its use in investigating the relationship between health and well-being and employee productivity. Environmental Health. 4, 1 (Jan. 2005), doi:10.1186/1476-069X-4-1

[11] Kroenke, K., Spitzer, R. L., and Williams, J. B. 2001. The PHQ-9: Validity of a Brief Depression Severity Measure. Journal of General Internal Medicine. 16, 9 (Sept. 2002), 606-613

[12] Martin, A., Rief, W., Klaiberg, A., and Braehler, E. 2006. Validity of the Brief Patient Health Questionnaire Mood Scale (PHQ-9) in the general population. General Hospital Psychiatry. 28, 1 (Feb. 2006), 71-77. DOI= 10.1016/j.genhosppsych.2005.07.003

[13] Bland JM, Altman DG. Cronbach's alpha. BMJ : British Medical Journal. 1997;314(7080):572. 